Discussion Despite difficulties to the practice of napping on the job, the promising results for both BP and BMI justify further investigations that could subsidise policies related to promoting adequate conditions for night workers to nap while on the job.

\section{$1602 f$ PRACTICAL GUIDELINES FOR LINKING BETTER WORKING TIME ARRANGEMENTS WITH STRESS PREVENTION AT WORK}

Kazutaka Kogi* ${ }^{\star}$ Y Sano. Ohara Memorial Institute for Science of Labour, Japan

\subsection{6/oemed-2018-ICOHabstracts. 1386}

Introduction Multifaceted work redesign is always involved in improving working time arrangements. Recent experiences in improving work systems with excessive work hours or overworking situations clearly show the need to link better working schedules with comprehensive measures to reduce stress at work. It is useful to know practical ways to facilitate joint changes or work schedules and job content.

Methods Typical types of improvements undertaken in participatory programmes for joint improvement of working time arrangements and job content for preventing stress at work were compared. The programmes studied included participatory occupational health activities of health care workers, local government employees and small enterprise workers. The common features of the participatory steps that facilitated the joint change process and the roles of trained facilitators were examined. The results were discussed to comile practical guidelines for linking better work schedules with other multifaceted stress-reducing improvements.

Results Multiple aspects addressed by the reviewed programmes commonly included team-based communication, work schedules, ergonomic work methods, physical environment and social support. Work schedule changes were usually combined with enhanced communication or improved work methods. It was found useful to utilise action-oriented tools such as action checklists reflecting local good practices and group work methods for proposing feasible improvements. New guidelines for organising participatory steps for the joint change of work schedules and job content were compiled with emphasis on simple group work procedures and the use of action-oriented checklists for proposing multifaceted actions.

Conclusions The participatory steps utilising action-oriented checklists and local good practices proved useful for facilitating planning and implementation of multifaceted improvements in work schedules and job content in the local context. It is suggested to organise participatory activities referring to the new guidelines compiling these positive features in linking working time arrangements and stress prevention at work.

\section{$1602 \mathrm{~g}$ SHIFTWORK, AUTOMATED VEHICLES, AND FUNCTIONAL IMPAIRMENTS IN TRANSPORTATION}

D Fisher*, S Popkin, M Lohrenz, K Davies-Schrils, D Moore. U.S. Department of Transportation, Volpe Centre, Cambridge, USA

\subsection{6/oemed-2018-ICOHabstracts. 1387}

Introduction Shiftwork has been linked to functional impairments such as fatigue and distraction that increase crash risk. Automated vehicles may decrease these risk factors. Simultaneously, automated vehicles will allow a broader range of 'drivers' to operate the vehicle such as those with cognitive and physical impairments, creating additional benefits and impacts to shiftworking drivers with such impairments. We have developed a conceptual model of how automation at all levels can be used to reduce the impacts of shiftwork on functional impairments in transportation.

Methods Over 100 articles were reviewed in the areas of shiftwork, automation, and functional impairment using established key words and recognised search domains. Central to the understanding of the relations among shiftwork, automation and functional impairments is knowledge of how drivers use, misuse, disuse and are abused by automation.

Results The result of the literature search and its analysis is a conceptual model which clarifies how shiftwork impacts the use, misuse and disuse of automation at each level, and how those impacts affect drivers with different types and levels of functional impairment. Existing research suggests that long shifts can lead to an increase in the misuse of automation, with fatigued and distracted drivers over-trusting automation. This effect will be magnified for those with cognitive impairments. However, systems are now deployed that can detect driver state such as fatigue and distraction, and could potentially communicate with an automatic driving suite and intervene when the driver is fatigued or distracted.

Discussion The conceptual model creates a roadmap for future research, applications and regulations that various stakeholders can use to improve the safety and well-being of shiftworkers.

\section{SHIFT WORK AS OCCUPATIONAL STRESS RISK FACTOR}

I Bukhtiyarov*, M Rubtsov. FSBSI 'Izmerov Research Institute of Occupational Health', Moscow, Russia

10.1136/oemed-2018-ICOHabstracts. 1388

The analyses of international and domestic publications shows that shift work is different professional groups occupational stress risk factor. From 2010 according IARC conclusion shift work is probable occupational cancer risk factor ('2A') primarily, breast cancer in women (nurses). The probability of association between shift work and increased of any other cancer and another occupations as well as connexion with frequency and duration of night shift work is discussed today.

There are bidirectional relationship between sleep disorders and work connected with psychosocial risks (style of work, high level of requirements and low level of control, working hours and shift mode, as well as imbalance between labour costs and remuneration Including). Desynchronosis in the shift mode, especially with rotation shifts, has negative stressful effect on physical and mental health, leading to increased risk of insomnia, restless leg syndrome, shift work sleep disorder, narcolepsy, cataplexy, obstructive sleep apnea syndrome, cardio-vascular disorders, metabolic syndrome, diabetes, and disorders of nervous system state and mental health.

The current hygienic classification in Russia makes it possible to assess the contribution of labour intensity as stress factor, including taking into account shifts. Analysis of shift work impact into risk of health disorders of law enforcement officers, doctors, nurses, railway workers data shows the dependence of its development probability on the of work experience with the rotation shifts, age and sex, primarily in the part of causal relationships between the work schedule and hypertension in conjunction with metabolic disorders development. 
The development of practical recommendations for shift work, the definition of duration and intensity of night shift rotation and shift schedules significance, taking into account the individual characteristics of workers (for example, the chronotype), requires further studies, as well as the issue of potential carcinogenic risk of shift work

\section{SHIFT WORK AND WORKPLACE VIOLENCE AGAINST HEALTHCARE WORKERS IN PSYCHIATRIC WARDS: A CASE-CONTROL STUDY}

G d'Ettorre ${ }^{1 *}, V$ Pellicani'. ' Local Health Authority of Brindisi (ASL BR), Department of Occupational Medicine, Italy

\subsection{6/oemed-2018-ICOHabstracts.1389}

${ }^{2}$ Local Health Authority of Lecce (ASL LE), Department of Mental Health, Italy

Introduction Workplace violence (WPV) towards healthcare workers (HCWs) employed in psychiatric wards (PWs) represeants a concern for healthcare organisations, globally. To date there is a lack of scientific data about the relationship between work-shifts and the occurrence of WPV against PW HCWs. The aim of the present study was to investigate the relationship between work shift schedules and WPV among registered nurses (RNs) working on non-traditional shifts, including nights and 12 hour shifts.

Methods The authors conducted a cross-sectional nested casecontrol analysis of data regarding the episodes of WPV perpetrated by patients or their relatives against RNs employed in two PWs, in the period between January-December 2016.

Results The one-year incidence of WPV was 31,50 per 100 Full Time Equivalent (FTE) positions. Cumulative nightshifts were significant for 3 or more nightshifts compared to working less than 3 nightshifts during the 7 days prior to the occurrence of WPV; additionally, RNs working 9 or more night-shifts showed higer risk of experiencing WPV compared to RNs working less than 4 night-shifts in the previous 28 days.

Discussion In the present study the occurrence of WPV against PW RNs was significantly correlated with shift- work; as consequence, the findings support the need of organisational interventions aimed at preventing the WPV and targeted on the management of shift-work schedules, with the aim of:

- limiting the night shifts up to two per week and up eight per month;

- adopting constant forward-rotating shift schedules.

\section{ASSOCIATION BETWEEN SOCIAL JETLAG AND OBESITY AMONG MALE FACTORY WORKERS IN JAPAN}

Reiko Kuroda*, Kenya Yamamoto, Yasushi Okubo. Division for Environment, Health and Safety, the University of Tokyo, Tokyo, Japan

\subsection{6/oemed-2018-ICOHabstracts. 1390}

Introduction Obesity is a major risk factors for noncommunicable diseases such as cardiovascular diseases, musculoskeletal disorders, and some cancers, and this is preventable. Not only sleep duration or chronotype have been associated with body mass index (BMI), but also circadian misalignment 'social jetlag' may be possibly associated in recent studies. We investigated whether social jetlag were associated with obesity, independent from sleep duration or chronotype.

Methods A cross-sectional study was conducted among 1357 male workers who works at an electrical manufacturer from April 2014 to January 2015. Body height and weight, smoking status and frequency of alcohol drinking were collected via annual medical check-up.

Sleep duration, chronotype, and other work- or life- related factor were collected through an additional questionnaire. Social jetlag (SJ) was calculated as the absolute difference between mid-sleep time on weekdays and weekends and were categorised into four groups. Logistic regression analysis was performed to estimate the odds ratio (OR) for 1) obesity $\left(B M I \geq 30 \mathrm{~kg} / \mathrm{m}^{2}\right.$ ) and 2) abdominal obesity (Waist circumference $\geq 90 \mathrm{~cm}$ ) for each SJ category (SJ $<1$ hour as reference) with adjustments for demographic, work- and life- related factors. A P value $<0.05$ was considered significant. Data were analysed using SPSS Ver.22.0.

Results Extreme SJ category ( $\leq 3$ hours) had significant high OR (95\% confidence interval [CI]) of 1) obesity and 2) central obesity after controlling for age, presence of night shift work, chronotype, sleep duration. OR (95\% CI) were 3.68 (1.49-9.11), and $2.48(1.28-4.79)$, respectively. We found no impact of SJ on BMI or abdominal obesity after controlling for additional other variables.

Conclusion It was found that extreme social jetlag possibly associated with high OR of obesity or abdominal obesity, independent from sleep duration and chronotype. However, there were no associations between social jetlag and BMI or abdominal obesity, when we considered other life-style variables.

\section{THE IMPACT OF SHIFTWORK ON SLEEP QUALITY AMONG NHS NURSES}

${ }^{1} \mathrm{~K}$ McDowall ${ }^{*}{ }^{1} \mathrm{E}$ Murphy, ${ }^{2} \mathrm{~K}$ Anderson. ${ }^{1}$ Occupational Health Service, Newcastle Hospitals NHS Foundation Trust, UK; ${ }^{2}$ Neurology Department, Newcastle Hospitals NHS Foundation Trust, UK

\subsection{6/oemed-2018-ICOHabstracts. 1391}

Introduction Shiftwork is common amongst nurses and it is known to be a workplace hazard as it may cause poor sleep quality, which can impact adversely on the health and safety of nurses and their patients. The aims of this study were to identify and describe the association between poor sleep quality and shiftwork in nurses. Additionally, to explore factors that contribute to poor sleep quality and to assess the awareness of support from Occupational Health $(\mathrm{OH})$.

Methods Cross-sectional study of nurses at a National Health Service Foundation Trust, February to March 2016. Data was collected via an online questionnaire. Sleep quality was measured using the Pittsburgh Sleep Quality Index.

Result 888 nurses participated; 34\% response rate. The prevalence of poor sleep quality was $78 \%$ (95\% CI: 0.748 to 0.813 ) in the shift working nurses (SWNs), compared to $59 \%$ (95\% CI: 0.503 to 0.678 ) in the non-shift working nurses (NSWNs). There was a mean sleep quality score difference of 1.58 between the SWNs and the NSWNs, which was statistically significant, $\mathrm{p}<0.001$ (95\% CI: 0.913 to 2.246). Undertaking shiftwork was the only significant association with poor sleep quality, when controlling for the other variables of age, gender and number of years worked, OR $0.410 \quad p<0.001$ (95\% CI: 0.265 to 0.634 ). 